\title{
Experiencias
}

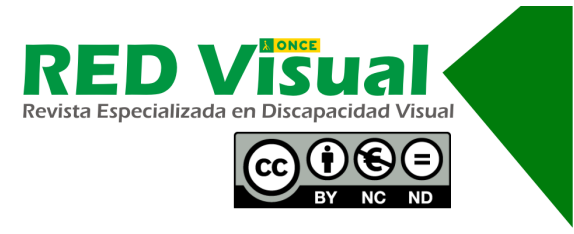

\section{Los Blindhéroes: ajuste a la discapacidad visual de alumnos de 3 a 10 años a partir de cuentos y muñecos personalizados ${ }^{1}$}

Blindheroes: adjustment to visual disability among 3- to 10-year-olds with personalised stories and dolls

M. J. Gómez Paredes, R. de Castro Maqueda

\section{Resumen}

El objetivo de esta experiencia es trabajar la autoestima y el ajuste al déficit visual de alumnos de 3 a 10 años con discapacidad visual, fomentando el aprendizaje de estrategias de afrontamiento. Esto se realiza a través de los personajes que se han elaborado y en los que creemos que se ven reflejados los niños. Nuestro alumnado presenta una dificultad añadida debido a su diversidad funcional visual. Creemos que podemos ayudarles a aceptarse como son, mejorando así sus relaciones sociales, valorando de forma adecuada sus capacidades, disfrutando de la mayor autonomía que su diversidad funcional les permita y consiguiendo todo aquello que se propongan. Elaborar un personaje y un cuento con sus propias características, adornadas con superpoderes (porque todos somos superhéroes), puede servir de base para comenzar el trabajo de aceptación de sus diferentes capacidades.

\section{Palabras clave}

Ajuste. Autoestima. Desarrollo. Diversidad funcional visual. Afrontamiento.

1 Trabajo galardonado con uno de los accésits del IV Concurso de Experiencias de Innovación y Buenas Prácticas en Servicios Sociales de la ONCE, presentado con el título Los Blindhéroes. 


\begin{abstract}
This experience was designed to help 3- to 10-year-old pupils with visual disability acquire self-esteem and adjust by encouraging them to learn coping strategies. It was based on the creation of two characters in whom children were believed to see themselves reflected. These pupils faced an added difficulty due to their visual diversity. The aim was to help them accept that reality to improve their social relationships, value their aptitudes appropriately, benefit from the greatest independence their functional diversity allows and reach all the goals they set themselves. Creating a character with superpowers (because we're all superheroes) and a story featuring their own experience may serve as a starting point for working on acceptance of their differing aptitudes.
\end{abstract}

\title{
Key words
}

Adjustment. Self-esteem. Development. Visual functional diversity. Coping.

\section{Presentación y justificación}

-Mamá, ¿por qué no hay un cuento en el que salga una niña como yo? ¿Por qué no hay una muñeca como yo?

A partir de esta pregunta nace el trabajo que se presenta: una madre preocupada porque su hija, con una prótesis ocular, no encontraba, entre todos sus cuentos y muñecos, un personaje con el que pudiera identificarse o compartir su «particularidad».

La madre se pone en contacto con su maestra de apoyo del Equipo Específico al alumnado con discapacidad visual de Sevilla (España) y, a partir de ahí, surge la idea de crear una pequeña colección de personajes y cuentos en relieve de niños con diferentes patologías visuales.

Evidentemente, trabajar la autoestima y el ajuste al déficit visual es la base fundamental del trabajo con los materiales que se presentan, fomentando el aprendizaje de estrategias de afrontamiento, es decir, enseñar a buscar estrategias cognitivas y conductuales para gestionar las demandas internas o externas que sean percibidas como excesivas y que les sirvan para enfrentarse a nuevos retos. Esto se realiza a través de los personajes que se han elaborado, en los que creemos que se verán reflejados. 
Este alumnado de 3 a 10 años presenta una dificultad añadida. Creemos que podemos ayudarles a aceptarse como son, mejorando así sus relaciones sociales, valorando de forma adecuada sus capacidades, disfrutando de la mayor autonomía que su diversidad funcional les permita y consiguiendo todo aquello que se propongan.

Sabemos que los cuentos son un instrumento ideal para afrontar estos temas tan importantes para un desarrollo óptimo de los menores, ya que mejoran sus diversas estrategias, su independencia y su relación con el entorno. Abrimos una ventana a que puedan desahogarse comunicándose.

En cuanto al juego:

Comúnmente se le identifica con diversión, satisfacción y ocio, con la actividad contraria a la actividad laboral, que normalmente es evaluada positivamente por quien la realiza. Pero su trascendencia es mucho mayor, ya que a través del juego se transmiten valores, normas de conducta, resuelven conflictos, educan a sus miembros jóvenes y desarrollan muchas facetas de su personalidad.

[...] Freud, por su parte, relaciona el juego con la necesidad de la satisfacción de impulsos instintivos de carácter erótico o agresivo, y con la necesidad de expresión y comunicación de sus experiencias vitales y las emociones que acompañan estas experiencias. El juego ayuda al hombre a liberarse de los conflictos y a resolverlos mediante la ficción. (López, 2010; pp. 18-19).

Los niños y niñas atendidos por los equipos de Sevilla y Cádiz, integrados en sus centros educativos, forman parte de un grupo en el que, a partir de esta experiencia con cuentos y muñecos personalizados, podemos educar en valores como la amistad, el respeto, la tolerancia y las normas de convivencia, a la vez que mejoramos el ajuste de los mismos.

\section{Objetivos}

Con la elaboración del material que se presenta, además del propio disfrute que ofrecen como cuentos y muñecos, se pretenden alcanzar varios objetivos:

- Dotar a nuestro alumnado de un material lúdico con el que se pueda identificar.

- Fomentar el juego simbólico, aprovechando todos los beneficios que este proporciona (desarrollo del lenguaje, favorecimiento de la asimilación y comprensión

Gómez, M.J., y Castro, R. de (2020). Los Blindhéroes: ajuste a la discapacidad visual de alumnos de 3 a 10 años a partir de cuentos y muñecos personalizados. RED Visual: Revista Especializada en Discapacidad Visual, 76, 62-74. https://doi.org/10.53094/KVRP5729. 
del entorno, representar situaciones mentales reales o imaginarias, desarrollo de la imaginación, desarrollo emocional, desarrollo psicomotor...).

- Favorecer el desarrollo social, los procesos de comunicación y cooperación con los demás.

- Facilitar el conocimiento de las diferencias individuales y las necesidades de las personas con diversidad funcional (en nuestro caso visual).

\section{Población destinataria de los materiales}

El trabajo realizado se ha inspirado en dos alumnas de Educación Infantil de segundo ciclo, en dos alumnos del primer ciclo de Educación Primaria y un alumno de Educación Especial (5 años).

Toda esta población está escolarizada en distintos centros educativos de las provincias de Cádiz y Sevilla.

Aunque la realización de los cuentos y muñecos está basada en esta población, se le ha presentado también a otro alumnado con características y dificultades parecidas a las de los modelos utilizados, aunque no estuvieran en el rango de edad elegido. Son alumnos que han podido beneficiarse de esta actividad dentro de su proceso de ajuste al déficit, bien por falta de madurez o bien porque no aceptaban del todo el uso de ayudas ópticas y/o no ópticas.

En la mayoría de los casos, la presentación de los cuentos y muñecos se ha realizado en el grupo-clase. Cuando no ha sido viable, se ha entregado el material al alumnado junto a su familia, explicándoles los objetivos del mismo y orientándoles en su uso.

\section{Temporalización y fechas de realización}

Durante el primer trimestre del curso 2018-2019 realizamos un estudio de la población que podía ser objetivo de este trabajo, es decir, alumnado de segundo ciclo de Educación Infantil y del primer ciclo de Primaria. Se seleccionaron las patologías más

Gómez, M.J., y Castro, R. de (2020). Los Blindhéroes: ajuste a la discapacidad visual de alumnos de 3 a 10 años a partir de cuentos y muñecos personalizados. RED Visual: Revista Especializada en Discapacidad Visual, 76, 62-74. https://doi.org/10.53094/KVRP5729. 
habituales entre ellos y las dificultades que estas podían presentar, además de las ayudas ópticas y no ópticas que podían utilizar.

Durante el segundo trimestre, nos centramos en la elaboración de todo el material que se presentaría: muñecos en fieltro y cuentos en relieve con los personajes seleccionados.

Durante el tercer trimestre, presentamos el material al alumnado, compañeros, centros educativos y familias, según los casos.

\section{Metodología}

Se han elaborado cinco personajes y sus correspondientes cuentos. Cada uno de ellos presenta una patología visual diferente (las más habituales entre nuestro alumnado).

En los cuentos se hace una pequeña presentación del personaje y de las ayudas ópticas y/o no ópticas que debe utilizar. A continuación, en la Tabla 1 se describe cada uno de los personajes.

Tabla 1. Los Blindhéroes

\begin{tabular}{|l|l|l|l|}
\hline Nombre & Patología & Artefacto & Superpoder \\
\hline $\begin{array}{l}\text { Celia: muñeca con trenzas, } \\
\text { un ojito pegado con velcro, } \\
\text { traje de bailarina y alas de } \\
\text { mariposa. }\end{array}$ & $\begin{array}{l}\text { Enucleación } \\
\text { de un ojo. }\end{array}$ & $\begin{array}{l}\text { Prótesis } \\
\text { ocular. }\end{array}$ & $\begin{array}{l}\text { Aumento de la } \\
\text { inteligencia. }\end{array}$ \\
\hline $\begin{array}{l}\text { Marcelo: muñeco con capa } \\
\text { roja, filtros protectores } \\
\text { y traje de superhéroe, rojo } \\
\text { y naranja, con la letra «M» } \\
\text { en el pecho. }\end{array}$ & Aniridia. & $\begin{array}{l}\text { Filtros } \\
\text { solares. } \\
\text { Atril. }\end{array}$ & $\begin{array}{l}\text { Evitar el deslum- } \\
\text { bramiento } \\
\text { Evitar problemas } \\
\text { de espalda. }\end{array}$ \\
\hline
\end{tabular}

Gómez, M.J., y Castro, R. de (2020). Los Blindhéroes: ajuste a la discapacidad visual de alumnos de 3 a 10 años a partir de cuentos y muñecos personalizados. RED Visual: Revista Especializada en Discapacidad Visual, 76, 62-74. https://doi.org/10.53094/KVRP5729. 


\begin{tabular}{|c|c|c|c|}
\hline Nombre & Patología & Artefacto & Superpoder \\
\hline $\begin{array}{l}\text { Marina: muñeca con pelo } \\
\text { rizado, bastón blanco, línea } \\
\text { braille colgada y letra «M» } \\
\text { en braille en el pecho. } \\
\text { Capa morada. }\end{array}$ & Ceguera total. & $\begin{array}{l}\text { Bastón } \\
\text { blanco. } \\
\text { Línea braille. }\end{array}$ & $\begin{array}{l}\text { Desplazarse por } \\
\text { cualquier lugar } \\
\text { sin tropezar } \\
\text { y en oscuridad. } \\
\text { Mandar mensajes } \\
\text { a todo el mundo. }\end{array}$ \\
\hline $\begin{array}{l}\text { Anthony: muñeco con } \\
\text { implante coclear, bastón } \\
\text { rojo y blanco, letra «A» } \\
\text { en el pecho en braille y } \\
\text { LSE; capa verde y guantes } \\
\text { blancos. }\end{array}$ & Sordoceguera. & $\begin{array}{l}\text { Guantes } \\
\text { blancos. } \\
\text { Implante } \\
\text { coclear. } \\
\text { Bastón rojo } \\
\text { y blanco. }\end{array}$ & $\begin{array}{l}\text { Comunicarse sin } \\
\text { hablar. Desplazarse } \\
\text { sin tropezar } \\
\text { y en la oscuridad. }\end{array}$ \\
\hline $\begin{array}{l}\text { Lola: muñeca albina, con } \\
\text { gorro y pantalla protectora, } \\
\text { sol en el pecho. }\end{array}$ & Albinismo. & $\begin{array}{l}\text { Gorro. } \\
\text { Filtros. }\end{array}$ & $\begin{array}{l}\text { Pantalla protectora } \\
\text { y filtros contra los } \\
\text { rayos malignos del } \\
\text { sol. }\end{array}$ \\
\hline
\end{tabular}

Figura 1. Alumnas haciendo uso del cuento de Celia

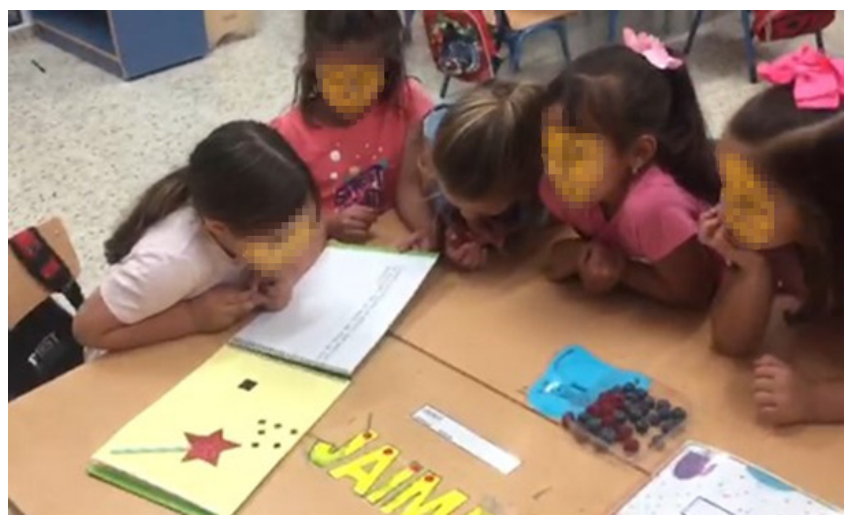

No siempre se presentan los cuentos y su personaje de la misma forma:

- Una vez que el maestro de apoyo ha trabajado individualmente con el alumno en cuestión, este presenta los materiales a sus compañeros de clase.

Gómez, M.J., y Castro, R. de (2020). Los Blindhéroes: ajuste a la discapacidad visual de alumnos de 3 a 10 años a partir de cuentos y muñecos personalizados. RED Visual: Revista Especializada en Discapacidad Visual, 76, 62-74. https://doi.org/10.53094/KVRP5729. 
- En los casos en los que lo anterior no fue posible (grave problema de ajuste, distanciamiento de las atenciones, absentismo escolar, enfermedad...), se le presenta al alumnado de forma individual y se orienta a la familia acerca de cómo utilizarlo.

Figura 2. Alumna con los cuentos y sus personajes

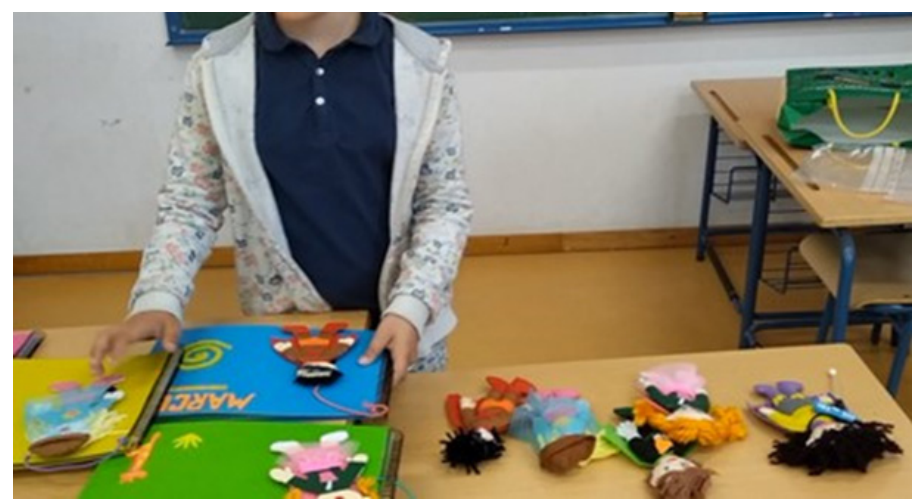

\section{Resultados}

Una vez llevada a cabo la presentación de los personajes y su correspondiente cuento a los distintos alumnos seleccionados y a su grupo-clase, se ha constatado que estos han podido identificarse con el personaje, por sus dificultades visuales y por la forma en que aquellos han resuelto algunos de sus problemas, usando las ayudas necesarias.

La mayoría del alumnado ha reconocido como facilitadores los instrumentos que se les proporcionan y que han aprendido o están aprendiendo a utilizar (ayudas ópticas y no ópticas, como filtros, atriles...).

En ciertos casos, se ha observado que algunos alumnos que pensábamos que no presentaban dificultades, han mostrado alguna reticencia a reconocer que se benefician de estas ayudas, pero la identificación con los personajes ha hecho posible que se paren a reflexionar sobre cómo estas pueden beneficiarles.

A pesar de que parte de nuestro alumnado pertenece, desde hace varios cursos, a un mismo grupo escolar, los compañeros no se han planteado casi nunca por qué su compañero tiene esas dificultades y por qué utiliza esas «cosas». Ponerse en su situación a través de los personajes ha beneficiado la comprensión de las dificultades que presenta su compañero $y$, en muchas ocasiones, han sido sus «guardianes», reclamando el uso de las ayudas cuando se «le olvida usarlas». 
Pensamos que este trabajo es solo el inicio de otro más largo y enriquecedor. Creemos que iniciar esta concienciación en edades tan tempranas va a favorecer el ajuste al déficit de un modo más normalizado y sin ocasionar dificultades más graves.

\section{Conclusiones}

Dos son las conclusiones de mayor relevancia que se pueden extraer del trabajo realizado:

- Es básico comenzar a trabajar cuanto antes el ajuste al déficit. Como se ha señalado anteriormente, el camino a recorrer por el alumnado con discapacidad visual es largo y, a veces, complicado. Reconocer las dificultades es importante, pero también potenciar las capacidades que las compensan.

- Debemos continuar este proceso de ajuste, acompañando al alumnado con discapacidad visual. Para ello, entre otras cosas, pretendemos continuar con la elaboración del material, completando las diferentes patologías visuales que podemos abordar.

De este primer trabajo surge un proyecto de investigación que se llevará a cabo durante el curso 2019-2020 y posteriores, en el que solicitaremos a los propios alumnos que elaboren «su cuento» y «su personaje» con nuestra ayuda y con la de su familia.

\section{Referencias bibliográficas}

López, I. (2010). El juego en la Educación Infantil y Primaria [PDF]. Autodidacta, 1(3), 19-37.

María Jesús Gómez Paredes. Maestra de apoyo. Dirección de Zona de la ONCE en Cádiz. Calle Acacias, 5; 11007 Cádiz (España). Correo electrónico: mgpa@once.es.

Rocío de Castro Maqueda. Maestra de apoyo. Centro de Recursos Educativos de la ONCE en Sevilla. Calle Campo de los Mártires, 10; 41018 Sevilla (España).Correo electrónico: mrcm@once.es. 


\section{Apéndice}

\section{Material elaborado}

Como se ha comentado anteriormente, el material consta de:

- Cinco muñecos realizados en fieltro; son personajes independientes, con características semejantes a las del alumno elegido, tanto físicas como de patología visual.

- Cinco cuentos. Estos cuentos están realizados con un personaje plano, pero con elementos en relieve que representa el mismo muñeco, con un imán en su espalda para poder pegarlo en todas las hojas del cuento. Elegimos los elementos más significativos de la narración (gafas, ventanas, atril, ordenador...) y se elaboraron con diferentes materiales, fáciles de conseguir, goma EVA, cartón, plástico...

A continuación, se muestra cada uno de los cuentos.

\section{Celia, niña con prótesis ocular}

Figura 3. Cubierta del cuento Celia

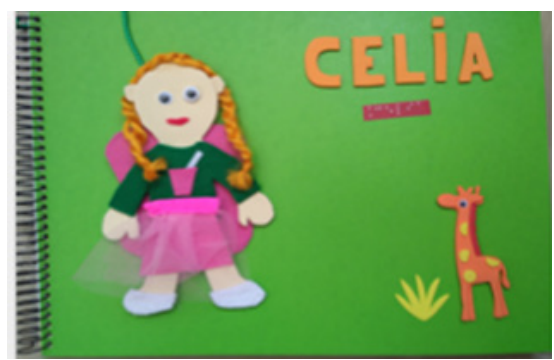

Tabla 2. Cuento de Celia

Hola. Me llamo Celia y soy una Blindhéroe.

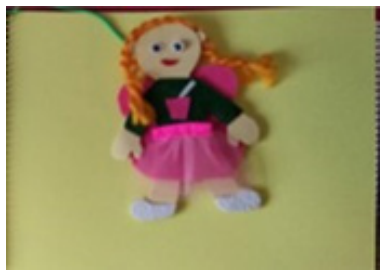

Cuando era pequeña, un médico, que creo que en realidad era un mago, me puso un ojo «mágico».
Yo no me di cuenta hasta que entré en el cole: ahí vi que sucedían cosas mágicas. Os contaré...

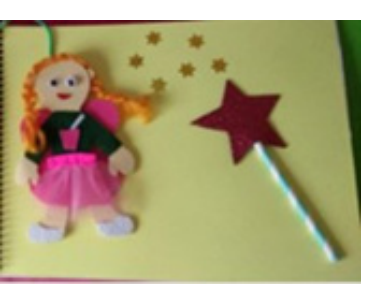


Descubrí que mi ojo «mágico» ayudaba al otro ojo a hacer las cosas superbién: leer, escribir, dibujar..., a ayudar a mis amigos.

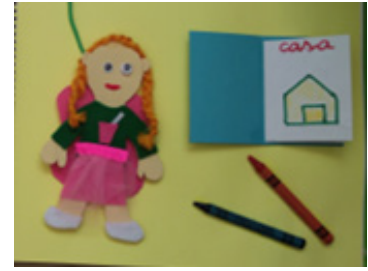

El ballet me encanta, y creo que no lo hago mal, aunque siempre se puede mejorar $y$, gracias a mi superpoder, sé que puedo hacer muchas cosas más.

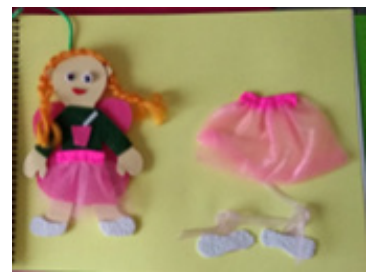

Un día seguro que conoceré a más amigos y amigas Blindhéroes como yo, y entonces, ipodremos mejorar el mundo!

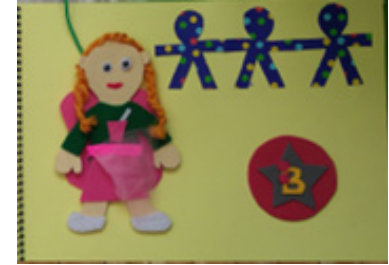

\section{Anthony, niño con sordoceguera}

Tabla 3. Cuento de Anthony

¿Qué tal? Soy Anthony, y vosotros no lo sabéis, pero ... soy un Blindhéroe.

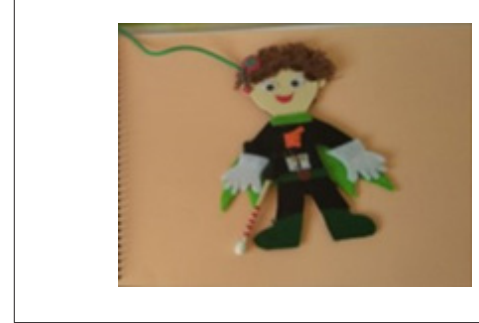

Con mis manos también descubro cómo son las cosas y las personas.

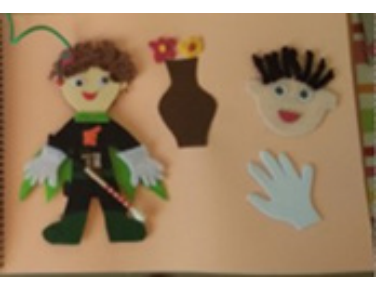

Tampoco sabéis que veo con mis manos y oigo con mi implante; iah!, y también uso mis manos para hablar.

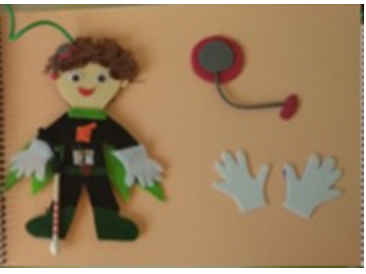

Uso un bastón rojo y blanco para ir de un sitio a otro sin tropezarme y para que las personas sepan que soy sordociego, además de Blindhéroe.
En el cole le enseño a mis compañeros cómo decir «hola, adiós...», todas las palabras, pero con las manos.

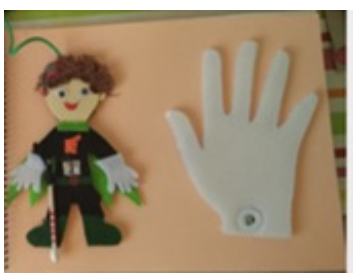

¿Sabéis si hay otros Blindhéroes? A mí me encantaría conocerlos.

Gómez, M.J., y Castro, R. de (2020). Los Blindhéroes: ajuste a la discapacidad visual de alumnos de 3 a 10 años a partir de cuentos y muñecos personalizados. RED Visual: Revista Especializada en Discapacidad Visual, 76, 62-74. https://doi.org/10.53094/KVRP5729. 
Figura 4. Cubierta del cuento Anthony

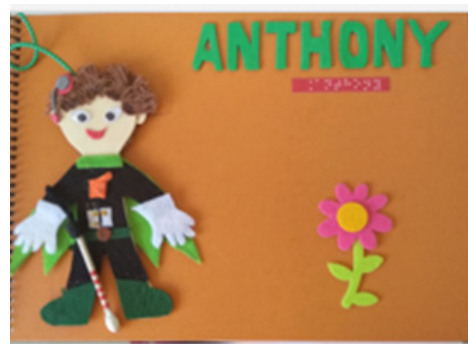

\section{Lola, niña con albinismo}

Figura 5. Cubierta del cuento Lola

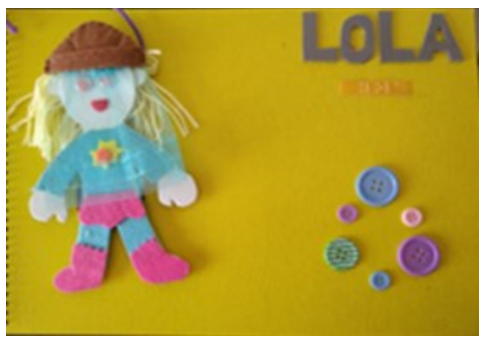

Tabla 4. Cuento de Lola

«Hola, Caracola». Me llamo Lola y soy una Blindhéroe.

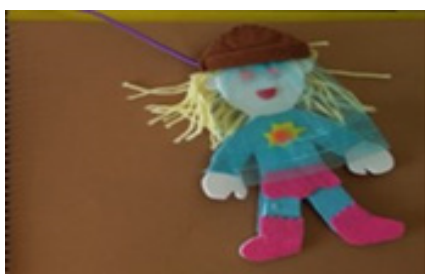

Cuando salgo al patio uso mis dos protecciones: mis filtros y mi sombrero.

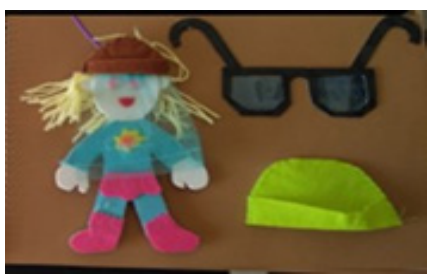

Me molesta mucho el sol en la piel y en los ojos, pero lo necesito, por eso he pensado llevarlo siempre en mi ropa.

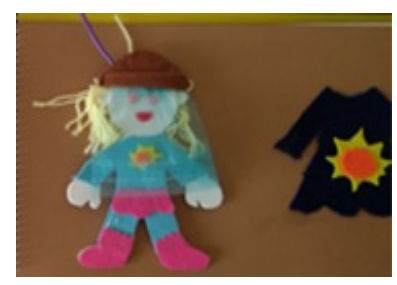

Cuando voy a la piscina cambio mis filtros por unas gafas para nadar; dentro del agua no me molesta la luz, solo el cloro cuando trago agua.

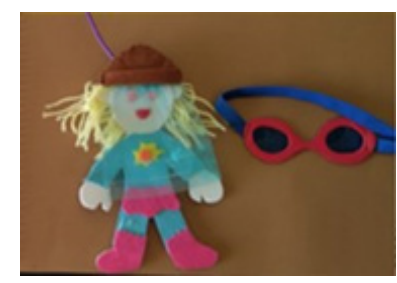

Mi seño en clase baja las persianas un poco para que no me moleste la luz.

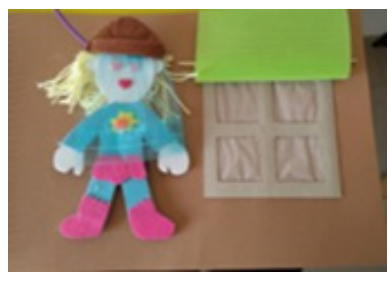

Mi madre me dice que hay más Blindhéroes como yo. ¿Dónde estarán?

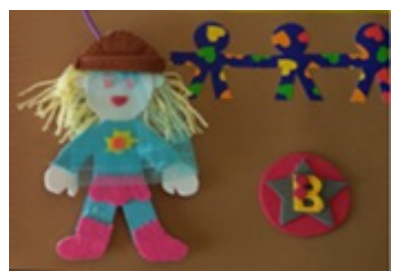

Gómez, M.J., y Castro, R. de (2020). Los Blindhéroes: ajuste a la discapacidad visual de alumnos de 3 a 10 años a partir de cuentos y muñecos personalizados. RED Visual: Revista Especializada en Discapacidad Visual, 76, 62-74. https://doi.org/10.53094/KVRP5729. 


\section{Marcelo, niño con aniridia}

Figura 6. Cubierta del cuento Marcelo

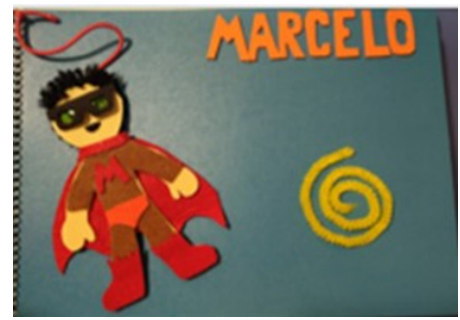

Tabla 5. Cuento de Marcelo

\begin{tabular}{|l|l|l|}
$\begin{array}{l}\text { Hola amigos, soy Marcelo y soy } \\
\text { un Blindhéroe. }\end{array}$ & $\begin{array}{l}\text { Me encanta jugar al fútbol en el } \\
\text { patio con mis compañeros. }\end{array}$ & $\begin{array}{l}\text { Para salir a la calle necesito } \\
\text { unos filtros, ya que la luz me } \\
\text { molesta mucho en los ojos. }\end{array}$ \\
\hline $\begin{array}{l}\text { En clase uso unas gafas para } \\
\text { leer y un atril para no «jorobar- } \\
\text { me», como los camellos. }\end{array}$ & $\begin{array}{l}\text { Susa, mi seño de la once, me } \\
\text { ha dicho que me va a poner } \\
\text { en el ordenador un programa } \\
\text { mágico para aprender a escri- } \\
\text { bir muy rápido. iMe encanta el } \\
\text { Mekanta! }\end{array}$ & $\begin{array}{l}\text { El otro día en el parque vi a un } \\
\text { niño que se parecía a mí, segu- }\end{array}$ \\
\hline \multirow{2}{*}{ rotro Blindhéroe. }
\end{tabular}

\section{Marina, niña ciega}

Figura 7. Cubierta del cuento Marina

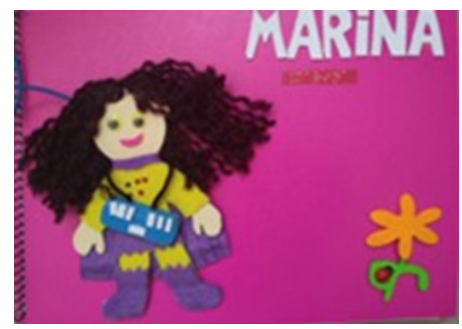

Gómez, M.J., y Castro, R. de (2020). Los Blindhéroes: ajuste a la discapacidad visual de alumnos de 3 a 10 años a partir de cuentos y muñecos personalizados. RED Visual: Revista Especializada en Discapacidad Visual, 76, 62-74. https://doi.org/10.53094/KVRP5729. 
Tabla 6. Cuento de Marina

\begin{tabular}{|c|c|c|}
\hline $\begin{array}{l}\text { iAh! Hola, no me había dado } \\
\text { cuenta de que estabais ahí. } \\
\text { Soy Marina. }\end{array}$ & $\begin{array}{l}\text { Tengo un poder especial: pue- } \\
\text { do ver con mis manos, aunque } \\
\text { no con mis ojos, pero no pasa } \\
\text { nada; por eso soy una Blindhé- } \\
\text { roe. }\end{array}$ & $\begin{array}{l}\text { Me encanta leer en braille, me } \\
\text { encanta dibujar en relieve, me } \\
\text { encanta..., uy, me encantan } \\
\text { muchas cosas. }\end{array}$ \\
\hline $\begin{array}{l}\text { Me muevo sola por el cole } \\
\text { usando mi bastón; bueno, y } \\
\text { también mis manos, siguiendo } \\
\text { las paredes. }\end{array}$ & $\begin{array}{l}\text { Pero hay una cosa que ahora } \\
\text { me gusta muuuuucho: mi } \\
\text { línea braille, con ella puedo } \\
\text { hacer cosas fantásticas. }\end{array}$ & $\begin{array}{l}\text { La verdad es que sé que hay } \\
\text { otros niños como yo, iestoy } \\
\text { deseando conocerlos! }\end{array}$ \\
\hline
\end{tabular}

Gómez, M.J., y Castro, R. de (2020). Los Blindhéroes: ajuste a la discapacidad visual de alumnos de 3 a 10 años a partir de cuentos y muñecos personalizados. RED Visual: Revista Especializada en Discapacidad Visual, 76, 62-74. https://doi.org/10.53094/KVRP5729. 